\section{Efficacy comparison of oseltamivir alone and oseltamivir-antibiotic combination for early resolution of symptoms of severe influenza-A and influenza-B hospitalized patients}

\author{
Azfar A. Ishaqui, Pharm-D, M.Phil, \\ Amer H. Khan, MS, PhD, \\ Syed A. Sulaiman, MS, PhD, \\ Muhammad Alsultan, Pharm-D, MS, \\ Irfanullah Khan, Pharm-D, MS.
}

\begin{abstract}
Objectives: To assess the comparative efficacy of oseltamivir alone and oseltamivir-antibiotic therapy for early relief of symptoms associated with severe influenza-A (non-H1N1) and influenza-B infection hospitalized patients.
\end{abstract}

Methods: In this retrospective multicenter study conducted from 2016-2019, enrolled patients were divided into 2 treatment groups. Group 1 patients were started on Antiviral drug (oseltamivir) alone therapy. Group 2 patients were initiated on Antiviral drug (oseltamivir) in combination with Antibiotic therapy. Using acute respiratory illness scoring, symptom severity score was assessed daily for 8 symptoms namely, fever, fatigue, headache, cough, sore throat, wheezing, muscle ache and nasal congestion. For each symptom the severity was scored from scale $0-3$.

Results: Overall mean ARI severity score was statistically significantly lower $(p<0.05)$ on day 2 (14.65-vs-13.68), day 3 (12.95-vs-11.67) and day 4 (10.31-vs-9.12) for influenza-A (non-H1N1) while day 3 (12.52-vs-11.87) and day 4 (11.21-vs-10.18) for influenza-B patients for patients who were initiated on oseltamivir-antibiotic combination therapy. Fever, cough and nasal congestion showed statistically significant improvement within 4 days of initiation of combination treatment. Fatigue, sore throat and muscle ache improvement pattern was same for both treatment protocols.

Conclusion: Oseltamivir-antibiotic combination treatment showed early resolution of some symptoms with cumulatively reduced mean symptom severity score in severe influenza infection hospitalized patients.
Keywords: oseltamivir, anti-bacterial agent, antiviral agents, symptom assessment, influenza-B virus

Saudi Med J 2020; Vol. 41 (9): 1015-1021 doi: 10.15537/smj.2020.9.25249

$\mathrm{I}$ nfluenza infection is still considered a threat as it is reported to affect approximately $20 \%$ of the world population every year during winter season. ${ }^{1}$ For prevention of seasonal influenza infection, trivalent, or quadrivalent inactivated vaccine is the only viable option available and antiviral drugs chemoprophylaxis therapy should not be used as a tool to replace seasonal influenza vaccine for prevention of influenza infection. ${ }^{2}$ It is usually recommended to initiate the treatment of antiviral drug oseltamivir (neuraminidase enzyme inhibitor) within 48 hours of onset of symptoms (ideally within 12 hours) for confirmed diagnosis. ${ }^{3}$ Some recent studies reported beneficial effects of oseltamivir even it started after beyond 48 to 72 hours after symptoms onset. ${ }^{4}$ Patients with severe influenza infection are usually presented in the hospital after 48 hours after the onset of symptom in which neuraminidase inhibitors are considered to be less effective. So, the need of time is to initiate neuraminidase inhibitors in combination with different class of drug for management of severe influenza infection. ${ }^{5}$ Oseltamivir combination therapy with clarithromycin and naproxen proved to be effective in lowering 30-day mortality as well as shorter hospitalization stay with less high dependency unit admission rate. ${ }^{6}$ A randomized clinical trial assessing the oseltamivir-azithromycin combination therapy reported early resolution and rapid recovery of some symptoms associated with severe influenza infection. ${ }^{7,8}$

The aim of current study was to assess the comparative efficacy of combination therapy of antiviral drug alone and antiviral-antibiotic combination therapy in early resolution of symptoms associated with severe infected patients with confirmed diagnosis of influenza-A (non-H1N1) and influenza-B hospitalized patients.

Methods. This multi-center record-based retrospective cohort study was conducted in 2 military hospitals of Saudi Arabia responsible to provide the health-care facilities to military personnel's and families of hospital staff. The duration of study was around 3 years starting from January 2016, until mid of 2019.

Approval of the study was obtained from King Abdullah Medical \& Research Center, Al-Ahsa, Saudi Arabia (RYD-18-417780-131817) and all patients given informed consent where applicable and patient confidentiality was maintained at all times as per 
Helsinki Declaration.

As per hospital protocol, all the patients who were found positive for influenza-like illness were assumed suspected for the influenza infection, and clinical samples (nasopharyngeal swabs, buccal swabs or nasal swabs) of patients were immediately sent to laboratory detection of influenza virus. Qualitative detection analysis of influenza virus strain(s) was carried out by using reverse transcriptase polymerase chain reaction (RT-PCR) technique. According to the study protocol, only patients who were detected positive for influenza-A (non-H1N1) strain and influenza-B strain considered eligible for study. All the influenza positive patients who were discharged from outpatient clinics or required hospitalization of less than 96 hours were excluded from the study. As per study protocol, influenza positive patients with severe illness who required hospitalization stay for duration of at least 96 hours (4-days) for management of influenza associated complications and symptoms were included in the study.

All identified patients of both influenza strains were separately classified into 2 treatment groups. The classification was carried out on the basis of prescribed drug therapy for initial 3-days (72 hours) of hospitalization soon after detection of influenza virus strain. Group 1 comprised of patients which were started on antiviral-monotherapy (oseltamivir) alone. Whereas, Group 2 included patients which were initially started on antiviral-combination therapy namely, oseltamivir and antibiotic (Cefuroxime, Ceftriaxone, Cefepime, Azithromycin, Meropenam or Vancomycin). The decision of antibiotic initiation and selection of antibiotic was merely prerogative of on-duty physician in consultation with infectious disease consultant based on the clinical status of patient, suspicion of secondary bacterial infection, comorbidity risk profile and symptoms severity.

Symptom severity was assessed by using acute respiratory illness (ARI) score. The scoring was usually carried out clinical round team's physician and nurse during the physician daily morning clinical round. The ARI score is based on assessing the severity of 8 common influenza symptoms; i) cough, ii) fatigue, iii) fever, iv) headache, v) muscle ache, vi) nasal congestion, vii) sore throat, and viii) wheezing. For each of 8 symptoms, a response is noted for each of 8 symptoms

Disclosure. Authors have no conflict of interests, and the work was not supported or funded by any drug company. on an ordinal scale of 0-3 namely, 0 -score $=$ absence of symptom, 1 -score $=$ mild symptom, 2 -score $=$ moderate symptom, 3 -score $=$ severe symptom. Total cumulative ARI score (range: 0-24) along with improvement in each of 8 symptoms score on daily basis were analyzed and compared for both group patients of influenza-A (non-H1N1) and influenza-B. ${ }^{8}$

Statistical analysis. Continuous variables such as age, weight, and so forth were mentioned in tables and results as mean value plus/minus standard deviation values and univariate analysis was performed using Mann-Whitney-U test/unpaired student T-test. Statistical significance was established at p-value of $<0.05$ considered as statistically significant. Univariate analysis of mean ARI score at different time intervals (every 24 hours) was also analyzed using unpaired student t-test. Statistical analysis was carried out by using softwares namely, GraphPad Prism, Version 5.1 and Statistical Package for Social Sciences Windows, version 16 (Chicago, SPSS Inc.).

Results. According to study protocol and inclusion criteria, 683 hospitalized patients were identified from which 399 were diagnosed from influenza-A (non-H1N1) strain infection and 284 patients were diagnosed influenza-B strain infection. Among influenza-A (non-H1N1) patients, 212 patients were administered oseltamivir alone (Group A1) and 187 patients were started on oseltamivir-antibiotic combination therapy (Group A2). For influenza-B patients, 153 patients were started on monotherapy namely, oseltamivir alone (Group B1) while 131 patients were started on oseltamivir-antibiotic combination therapy (Group B2).

Among demographic characteristics such as mean age and gender distribution, no significant difference was reported for both influenza strains patients separately. Enrolled patients were observed to be largely unvaccinated with only 98 (14\%) patients received current season influenza vaccine. Chronic respiratory illnesses were most common associated comorbidity. Demographic characteristics along with clinical features of patients at the time of hospital admission are mentioned in Table 1.

For influenza-A (non-H1N1) patients, cumulative mean ARI severity score was observed to be statistically significant less for combination treatment group and was statistically significant less on hospitalization day 2 (14.65 vs $13.68 ; p<0.001)$, day 3 (12.95 vs $11.67 ; p<0.001)$ and day 4 (10.31 vs $9.12 ; p<0.001)$ in univariate analysis. Univariate analysis of mean ARI severity scores for influenza-A (non-H1N1) patients are 
Antiviral-antibiotic in influenza-A \& B ... Ishaqui et al

Table 1 - Patient characteristics and clinical features of influenza-A and influenza-B patients at time of admission.

\begin{tabular}{|c|c|c|c|c|c|c|}
\hline \multirow[t]{2}{*}{ Patient characteristics } & \multicolumn{3}{|c|}{ Influenza-A (non-H1N1) } & \multicolumn{3}{|c|}{ Influenza-B } \\
\hline & $\begin{array}{c}\text { Group-A1 } \\
\text { (oseltamivir } \\
\text { alone) }\end{array}$ & $\begin{array}{c}\text { Group-A2 } \\
\text { (oseltamivir + } \\
\text { antibiotic) }\end{array}$ & $P$-value & $\begin{array}{c}\text { Group-B1 } \\
\text { (oseltamivir } \\
\text { alone) }\end{array}$ & $\begin{array}{c}\text { Group-B2 } \\
\text { (oseltamivir + } \\
\text { antibiotic) }\end{array}$ & $P$-value \\
\hline Enrolled patients & 212 & 187 & & 153 & 131 & \\
\hline \multicolumn{7}{|l|}{ Gender } \\
\hline Male & $99(46.7)$ & $90(48.1)$ & 0.78 & $72(47.1)$ & $61(46.5)$ & 0.93 \\
\hline \multicolumn{7}{|l|}{ Age } \\
\hline Mean \pm SD & $57 \pm 15.6$ & $55 \pm 17.2$ & 0.23 & $58.1 \pm 16.4$ & $57.5 \pm 14.3$ & 0.74 \\
\hline \multicolumn{7}{|l|}{ Influenza vaccinated patients } \\
\hline Yes (\%) & $31(14.6)$ & $24(12.8)$ & 0.61 & $25(16.3)$ & $18(13.7)$ & 0.37 \\
\hline \multicolumn{7}{|l|}{ Comorbidities } \\
\hline Respiratory diseases & $68(32.1)$ & $67(35.8)$ & 0.62 & $55(35.9)$ & $44(33.6)$ & 0.82 \\
\hline Endocrine disorders & $50(23.6)$ & $45(24.1)$ & 0.91 & $40(26.4)$ & $37(28.3)$ & 0.74 \\
\hline Cardiovascular diseases & $19(8.9)$ & $13(6.9)$ & 0.54 & $9(5.8)$ & $6(4.5)$ & 0.62 \\
\hline Hypertensive heart disease & $65(30.6)$ & $59(31.5)$ & 0.85 & $41(26.7)$ & $39(29.8)$ & 0.58 \\
\hline Renal disorders & $30(14.2)$ & $31(16.7)$ & 0.51 & $13(8.5)$ & $14(10.7)$ & 0.4 \\
\hline \multicolumn{7}{|l|}{ ER presenting complains } \\
\hline Fever & $157(74.1)$ & $146(78.1)$ & 0.35 & $106(69.2)$ & $94(71.8)$ & 0.65 \\
\hline Cough & $135(63.7)$ & $111(59.3)$ & 0.37 & $98(64.1)$ & $86(65.7)$ & 0.78 \\
\hline Nasal congestion & $121(57.1)$ & $114(61.0)$ & 0.43 & $75(49.2)$ & $69(52.6)$ & 0.54 \\
\hline Sore throat & $115(54.2)$ & $99(52.9)$ & 0.87 & $72(47.2)$ & $58(44.3)$ & 0.63 \\
\hline Dizziness & $79(37.2)$ & $73(39.1)$ & 0.72 & $49(32.0)$ & $46(35.1)$ & 0.58 \\
\hline Muscle pain & $59(27.8)$ & $49(26.2)$ & 0.71 & $47(30.7)$ & $44(33.5)$ & 0.61 \\
\hline Altered mental illness & $38(17.9)$ & $41(21.9)$ & 0.69 & $29(18.9)$ & $28(21.3)$ & 0.62 \\
\hline
\end{tabular}

Table 2 - Univariate analysis of cumulative mean acute respiratory illness (ARI) severity score for both group patients of influenza-A and influenza-B.

\begin{tabular}{|c|c|c|c|c|c|c|}
\hline \multirow[t]{2}{*}{ Days } & \multicolumn{6}{|c|}{ Influenza-A (non-H1N1) } \\
\hline & $\begin{array}{c}\text { Group A1 } \\
\text { (oseltamivir } \\
\text { alone) }\end{array}$ & $\begin{array}{c}\text { Group-A2 } \\
\text { (oseltamivir + } \\
\text { antibiotic) }\end{array}$ & $\begin{array}{c}\text { Difference between } \\
\text { means }\end{array}$ & $95 \%$ CI of diff. & $t$-value & $P$-value \\
\hline Day 1 & $15.66 \pm 2.98$ & $15.29 \pm 2.91$ & $0.37 \pm 0.29$ & $-0.21-0.94$ & 1.251 & 0.212 \\
\hline Day 2 & $14.65 \pm 2.63$ & $13.68 \pm 2.18$ & $0.97 \pm 0.24$ & $0.49-1.44$ & 3.980 & $<0.001^{*}$ \\
\hline Day 3 & $12.95 \pm 2.33$ & $11.67 \pm 2.30$ & $1.28 \pm 0.23$ & $0.82-1.73$ & 5.509 & $<0.001^{*}$ \\
\hline Day 4 & $10.31 \pm 2.20$ & $9.12 \pm 2.14$ & $1.19 \pm 0.21$ & $0.76-1.61$ & 5.461 & $<0.001^{*}$ \\
\hline \multirow[t]{2}{*}{ Days } & \multicolumn{6}{|c|}{ Influenza-B } \\
\hline & $\begin{array}{l}\text { Group B1 } \\
\text { (oseltamivir } \\
\text { alone) }\end{array}$ & $\begin{array}{c}\text { Group-B2 } \\
\text { (oseltamivir + } \\
\text { antibiotic) }\end{array}$ & $\begin{array}{c}\text { Difference between } \\
\text { means }\end{array}$ & $95 \%$ CI of diff. & $t$-value & $P$-value \\
\hline Day 1 & $14.26 \pm 2.89$ & $14.22 \pm 2.79$ & $0.04 \pm 0.33$ & $-0.62-0.70$ & 0.118 & 0.906 \\
\hline Day 2 & $13.74 \pm 2.39$ & $13.24 \pm 2.40$ & $0.50 \pm 0.28$ & $-0.06-1.05$ & 1.754 & 0.081 \\
\hline Day 3 & $12.52 \pm 2.38$ & $11.87 \pm 2.29$ & $0.65 \pm 0.27$ & $0.14-1.19$ & 2.335 & $0.020^{*}$ \\
\hline Day 4 & $11.21 \pm 2.31$ & $10.18 \pm 2.25$ & $1.03 \pm 0.27$ & $0.4975-1.56$ & 3.791 & $<0.001^{*}$ \\
\hline \multicolumn{7}{|c|}{ *significant, CI: confidence interval } \\
\hline
\end{tabular}


summarized in Table 2.

Univariate analysis for influenza-B patients showed that mean ARI symptom severity score was less for combination treatment group patients on treatment day 3 (12.52 vs $11.87 ; p=0.020)$ and day 4 (11.21 versus $10.18 ; p<0.001)$ of hospitalization. Univariate analysis of mean ARI severity scores for influenza-B patients are summarized in Table 2.

Comparison of 8 individual symptoms (cough, fatigue, fever, headache, muscle ache, nasal congestion, sore throat, and wheezing) among both treatment protocols at different time intervals namely: i) at time of admission, ii) day, 2 (iii) day 3, and (iv) day 4 are summarized in Figures 1 \& 2 for influenza-A
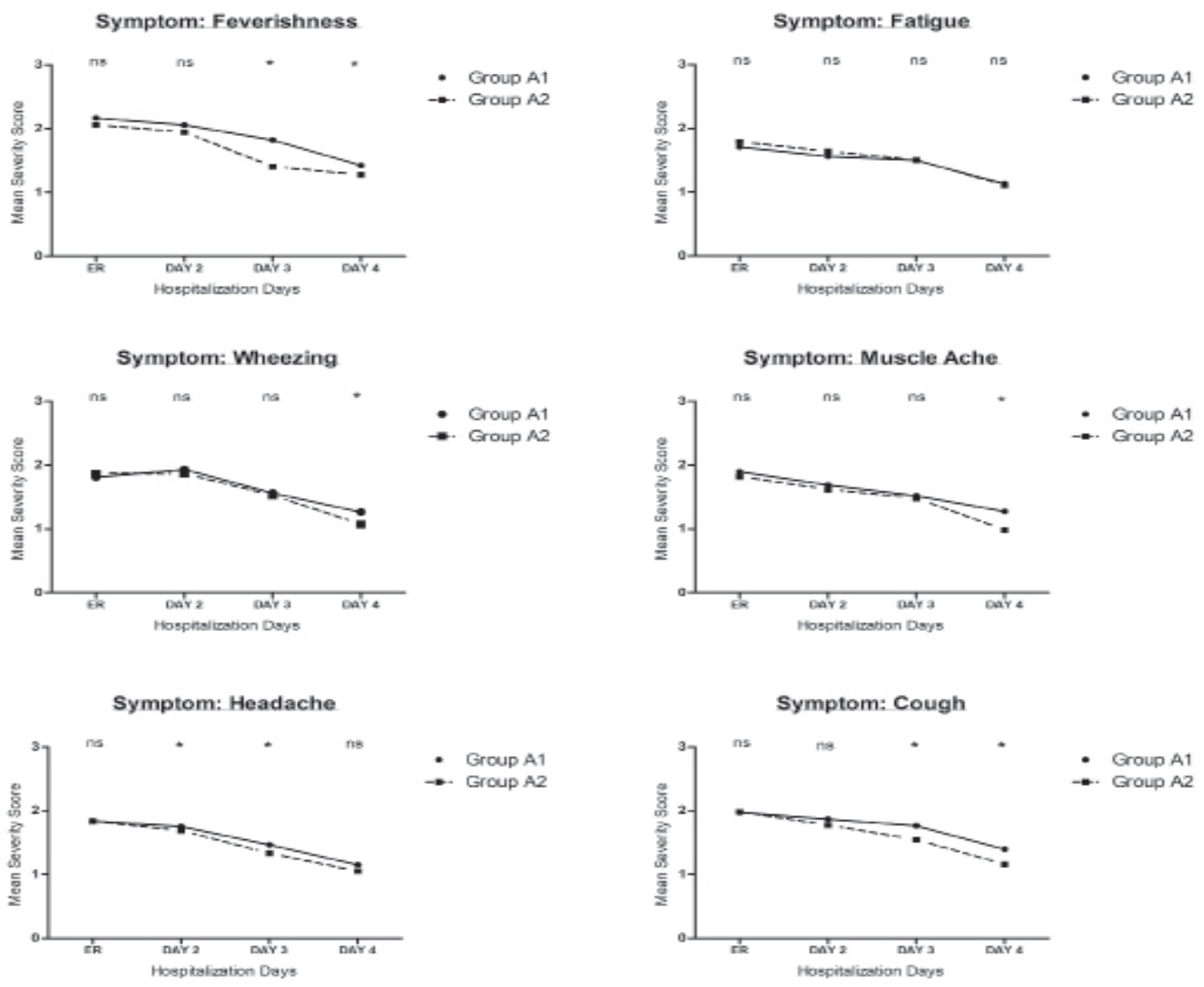

Symptom: Nasal Congestion

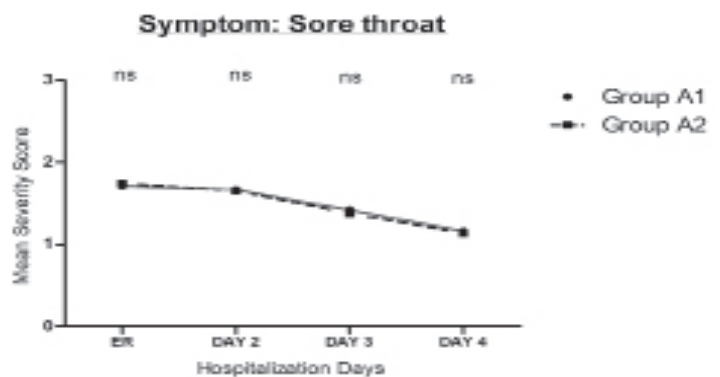

Figure 1 - Comparison of symptom severity of individual symptoms of Group A1 (oseltamivir alone) versus Group A2 (oseltamivir + antibiotic) for influenza-A patients. ER - emergency room 
(non-H1N1) and influenza-B patients. Except headache symptom, no symptom showed statistically significant improvement on treatment day 2 for Group A2 patients of influenza-A (non-H1N1) patients while no symptom showed statistically significant improvement on day 2 for influenza-B patients. Statistically significant improvement in fever was evident on day 3 and day 4 for combination treatment group patients of influenza-A and influenza-B patients.

Discussion. The current study was aimed to assess the comparative efficacy comparison of 2 drug
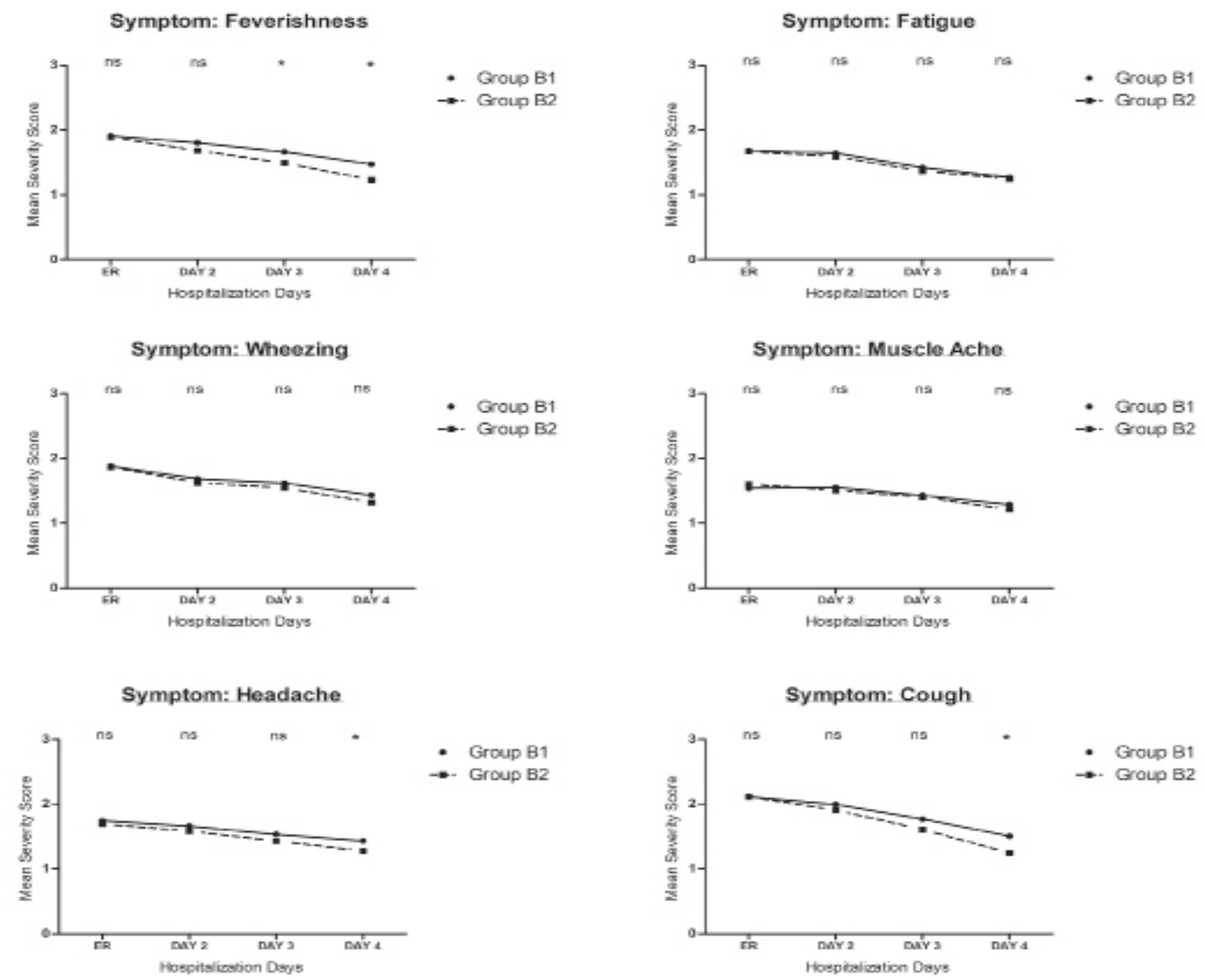

Symptom: Nasal Congestion

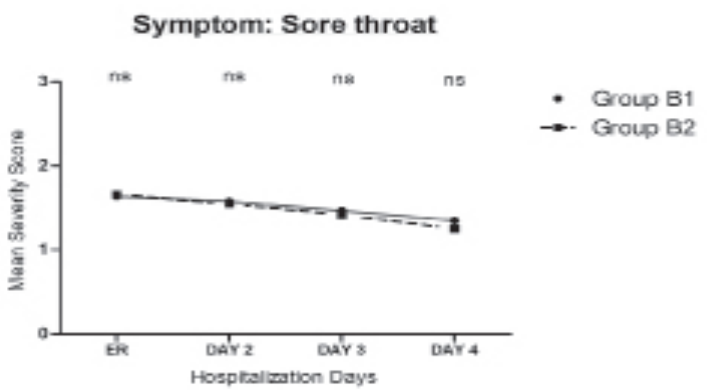

Figure 2 - Comparison of symptom severity of individual symptoms of Group B1 (oseltamivir alone) vs Group B2 (oseltamivir + antibiotic) for influenza-B patients. ER - emergency room 
therapies namely antiviral drug alone and antiviralantibiotic combination therapy aimed to analyze the rapidity of symptoms relief in severe influenza infection hospitalized patients by using ARI symptom severity score scale. Mean cumulative ARI score was observed to be statistically significant less in patients who received antiviral-antibiotic combination therapy as compared to patients who were started only on antiviral therapy alone. On hospitalization day 2, early resolution of symptoms was evident in influenza-A (non-H1N1) patients who received antiviral-antibiotic combination therapy because of statistically significant low mean cumulative ARI severity score of Group A2 patients. For influenza-B patients, early resolution of symptoms was statistically significant evident on treatment day 3 of hospitalization for patients who received antiviralantibiotic combination therapy.

Oseltamivir treatment has been proven effective in recovering from influenza illness both in terms of duration and severity in uncomplicated influenza patients. ${ }^{8}$ Previously random clinical trials have reported that the duration of symptoms were reduced by approximately one day if oseltamivir was initiated within 48 hours of influenza symptoms onset. ${ }^{3}$ One of the characteristics of influenza infection is the elevated levels of pro-inflammatory cytokines. ${ }^{9}$ Besides having antibacterial abilities, many antibiotics inhibits the production of pro-inflammatory cytokines specially macrolides and fluoroquinolones, which helps in reduction of cytokines level resulting in early resolution of some of the Influenza associated symptoms. ${ }^{10}$

In our current study, oseltamivir-antibiotic combination therapy was observed to be more efficacious than oseltamivir alone in rapidity of symptoms relief in both influenza types. Univariate analysis demonstrate that the mean cumulative ARI symptom score was statistically significant less for oseltamivir-antibiotic combination patients on day 2 , day 3 , and day 4 for influenza-A (non-H1N1) while day 3 and day 4 for influenza-B patients. Recent randomized clinical trial based on comparison of oseltamivir alone versus oseltamivir-azithromycin combination revealed that oseltamivir-azithromycin combination was associated with early resolution of symptoms of influenza infection patients along with reduced levels of pro-inflammatory cytokines. ${ }^{8,11}$

Fever was reported as one of the early symptoms in influenza patients in more than $50 \%$ of the influenza population. ${ }^{12}$ A meta-analysis reported that fever symptom is usually present in one-third of influenza infected patients. ${ }^{13}$ In our study, ratio of fever symptom present at early stage was high with 303 (75.9\%) patients of influenza-A (non-H1N1) and 200 (70.4\%) patients of influenza-B infection. A reason for this high ratio is because of the fact that in current study, all the study participants were hospitalized patients while the most of the previous studies included patients from outpatient settings. For oseltamivir-antibiotic combination patients, extremity of fever symptom was reduced statistically significantly on day 3 of initiation of treatment for both influenza-A (non-H1N1) and influenza-B. Symptoms such as fatigue and sore throat did not show any significant difference for both treatment protocols in influenza-A (non-H1N1) and influenza-B patients. Some symptoms such as muscle ache and wheezing showed statistically significant improvement on day 4 of treatment for oseltamivirantibiotic group patients of influenza-A (non-H1N1) while no statistically significant difference was found among both treatments of influenza-B patients.

In our current study, extremity of cough symptom was reduced gradually with both treatment protocols namely oseltamivir alone and oseltamivir-antibiotic combination therapy, but combination therapy proved to be more effective than oseltamivir alone therapy in early resolution of cough. For influenza-A (non-H1N1) patients, mean severity score of cough was statistically significantly less on day 3 of treatment for combination treatment group patients as compare to patients who received oseltamivir alone while statistically significant difference was evident on day 4 of treatment for combination treatment group influenza-B patients (Group-B2). ${ }^{14}$ Some studies reported that cough symptom can continue for more than 2 weeks in severe influenza infection. ${ }^{15}$ Thus, antiviral-antibiotic combination therapy can provide synergistic effect for prevention of influenza associated complications, severity of illness and early recovery of influenza infection symptoms.

Study limitations. An important limitation of the current study is failure to re-test confirmed influenza patients again for reconfirmation of detection of influenza strain because of possible variance between different specimens (nasal, nasopharyngeal or buccal swabs) used for detection of Influenza strain.

In conclusion, the enrolled population is largely unvaccinated with confirmed diagnosis of influenza-A (non-H1N1) and influenza-B hospitalized patients. Oseltamivir-antibiotic combination therapy has been found to be efficacious in reducing the overall mean ARI severity score on day 3 for influenza-A (non-H1N1) patients and on day 4 for influenza-B hospitalized patients. On comparison with oseltamivir alone, some symptoms such as cough and nasal congestion were statistically significantly improved from day 2 and day 3 
for influenza-A (non-H1N1) and influenza-B patients respectively. Severity of fever was significantly improved on day 3 for both types of influenza infection. Some symptoms such as fatigue, sore throat and muscle ache have not shown any difference between oseltamivirantibiotic combination and oseltamivir alone therapy.

Acknowledgment. We would like to thank Geeks Scientific Editing for their help in improving the language and proofreading services for current manuscript.

Received 15th May 2020. Accepted 6th July 2020.

From the Discipline of Clinical Pharmacy (Ishaqui, Khan A, Sulaiman, Khan I), School of Pharmaceutical Sciences, Universiti Sains Malaysia, Penang, Malaysia and from the Department of Pharmacy (Ishaqui, Alsultan), King Abdulaziz Hospital, National Guard Health Authority; and from the King Abdullah Medical Research Center (Ishaqui, Alsultan), Al Ahsa, Kingdom of Saudi Arabia.

Address correspondence and reprints request to: Dr. Amer H. Khan, Discipline of Clinical Pharmacy, School of Pharmaceutical Sciences, Universiti Sains Malaysia,Penang, Malaysia.E-mail:dramer@usm.my

ORCID ID: https://orcid.org/0000-0003-4802-6181

\section{References}

1. Rambaldi GZ, Giannotta M, Vocale C, Landini MP, Zompatori $\mathrm{M}$, editors. Chest $\mathrm{x}$-ray and HRCT findings in influenza $\mathrm{A} / \mathrm{B}$ pneumonia as a predictor of clinical course and intensive care hospitalisation. Austria: European Congress of Radiology; 2017.

2. Grohskopf LA, Alyanak E, Broder KR, Walter EB, Fry AM, Jernigan DB. Prevention and control of seasonal influenza with vaccines: recommendations of the Advisory Committee on Immunization Practices-United States, 2019-20 influenza season. MMWR Recommendations and Reports 2019; 68: 1.

3. Dai Z, Zhang L, Yu Q, Liu L, Yang M, Fan K. Early administration of oseltamivir within 48 hours after onset of flulike symptoms can reduce the risk of influenza B VirusAssociated Pneumonia in hospitalized pediatric patients with influenza B virus infection. Pediatr Infect Dis J 2020; 39: e20-e22.

4. Fry AM, Goswami D, Nahar K, Sharmin AT, Rahman M, Gubareva L, et al. Efficacy of oseltamivir treatment started within 5 days of symptom onset to reduce influenza illness duration and virus shedding in an urban setting in Bangladesh: a randomised placebo-controlled trial. Lancet Infect Dis 2014; 14: 109-118.

5. Beigel JH, Bao Y, Beeler J, Manosuthi W, Slandzicki A, Dar SM, et al. Oseltamivir, amantadine, and ribavirin combination antiviral therapy versus oseltamivir monotherapy for the treatment of influenza: a multicentre, double-blind, randomised phase 2 trial. Lancet Infect Dis 2017; 17: 1255-1265.

6. Hung IF, To KK, Chan JF, Cheng VC, Liu KS, Tam A, et al. Efficacy of clarithromycin-naproxen-oseltamivir combination in the treatment of patients hospitalized for influenza A (H3N2) infection: an open-label randomized, controlled, phase IIb/III trial. Chest 2017; 151: 1069-1080.

7. Kakeya H, Seki M, Izumikawa K, Kosai K, Morinaga Y, Kurihara $S$, et al. Efficacy of combination therapy with oseltamivir phosphate and azithromycin for influenza: a multicenter, open-label, randomized study. PLoS One 2014; 9: e91293.

8. Ishaqui AA, Khan AH, Sulaiman SAS, Alsultan MT, Khan I, Naqvi AA. Assessment of efficacy of oseltamivir-azithromycin combination therapy in prevention of influenza-a (H1N1) infection complications and rapidity of symptom relief. Expert Rev Respir Med 2020; 14: 533-541.

9. Chen S, Liu G, Hu A, Zhang L, Sun W, Tang W, et al. Ponatinib Protects Mice from Lethal Influenza Infection by Suppressing Cytokine Storm. Front Immunol 2019;10: 1393.

10. Kwiatkowska B, Maślińska M. Macrolide therapy in chronic inflammatory diseases. Mediators Inflamm 2012; 2012: 636157.

11. Lee N, Wong C-K, Chan MC, Yeung ES, Tam WW, Tsang OT, et al. Anti-inflammatory effects of adjunctive macrolide treatment in adults hospitalized with influenza: a randomized controlled trial. Antiviral Res 2017; 144: 48-56.

12. Falsey AR, Baran A, Walsh EE. Should clinical case definitions of influenza in hospitalized older adults include fever? Influenza Other Respir Viruses 2015; 9 Suppl 1: 23-29.

13. Carrat F, Vergu E, Ferguson NM, Lemaitre M, Cauchemez S, Leach S, et al. Time lines of infection and disease in human influenza: a review of volunteer challenge studies. Am J Epidemiol 2008; 167: 775-785.

14. Shaik AB, Prabhu M, Shenoy S, Thomson SR. Oseltamivirinduced neuropsychiatric symptoms. Journal of Pharmacology and Pharmacotherapeutics 2018; 9: 43.

15. Schmidt S. Complications of flu. SA Pharmaceutical Journal 2015; 82: 23-27. 